\title{
Die Nuklearwaffen der NATO. Das neue Strategische Kon- zept bietet die Chance einer Neuorientierung
}

\author{
Christos Katsioulis und Christoph Pilger*
}

\begin{abstract}
NATO needs to reconsider the role of nuclear weapons. The debates on a new Strategic Concept present an opportunity for a fundamental review, including the question of whether nuclear deterrence by NATO is still appropriate or has become counterproductive. NATO's contribution to stabilizing the non-proliferation regime should be an important concern in these discussions. A review of the political and military arguments leads to the conclusion that tactical nuclear weapons should be withdrawn from Europe and NATO should commit to a no first use nuclear policy.
\end{abstract}

Keywords: NATO, nuclear arms, Europe, strategy

NATO, Nuklearwaffen, Europa, Strategie

\section{Einleitung}

$\mathrm{D}$ ie NATO sieht sich im 60. Jahr ihres Bestehens einer Reihe von Herausforderungen gegenüber. Dabei stellt sich nicht zuletzt vor dem Hintergrund der wieder gespannteren Beziehungen zu Russland die Frage nach der zukünftigen Nuklearstrategie der NATO. Die NATO hat, spätestens für das Jahr 2010, die Ausarbeitung eines neuen Strategischen Konzepts angekündigt. Sie sollte sich in diesem Zusammenhang insbesondere mit dem Stellenwert der in Europa stationierten taktischen Nuklearwaffen auseinandersetzen. Dies gilt umso mehr, als die Haltung der NATO einen wesentlichen Impuls für die Zukunft des Nichtverbreitungsregimes geben dürfte. Die für das Jahr 2010 bevorstehende Überprüfungskonferenz zum nuklearen Nichtverbreitungsvertrag (NVV) ist aber nach dem Scheitern der Konferenz im Jahr 2005 sprichwörtlich zum „Erfolg verdammt“. Der vorliegende Beitrag stellt daher die Frage, welche Rolle Nuklearwaffen gegenwärtig für die NATO spielen, und welche Bedeutung sie in dem neuen Strategischen Konzept erlangen sollten.

\section{Nichtverbreitung und Abrüstung}

Atomare Rüstungskontrolle und Abrüstung befinden sich in einem Übergangszustand. Seit dem Ende des Ost-West-Konflikts sind die Nuklearwaffenarsenale zunächst in beeindruckendem Maße geschrumpft. Auf der Überprüfungskonferenz des Nichtverbreitungsvertrags im Jahr 2000 einigte man sich auf 13 Schritte zur Implementierung der Verpflichtungen aus Artikel VI des Abkommens. ${ }^{1}$

Nach diesen Fortschritten ist der Prozess der Rüstungskontrolle und Abrüstung zum Stillstand gekommen. Bei der letzten Überprüfungskonferenz des NVV im Jahre 2005 gab es keine Einigung. Der NVV ist das zentrale Element der Nichtverbrei-

\footnotetext{
* Christos Katsioulis ist Projektleiter für Nichtverbreitung und Abrüstung bei der Friedrich-Ebert-Stiftung, Berlin; Christoph Pilger ist wissenschaftlicher Mitarbeiter von Uta Zapf, MdB, Berlin. Die Autoren danken Hansfrieder Vogel und Helmut Hugler für ihre Kritik und Unterstützung.

1 Im Gegenzug für die Verpflichtung der Nichtatomwaffenstaaten, auf die Entwicklung von Atomwaffen zu verzichten, sagten die Atomwaffenstaaten zu, ihre Arsenale abzubauen.
}

tung und eine tragende Säule der globalen Sicherheitsarchitektur, Stillstand bietet daher Grund zur Beunruhigung. Bezeichnenderweise gibt es alarmierende Tendenzen hin zu einer Weiterverbreitung von Atomwaffen: 1998 testeten Indien und Pakistan Nuklearwaffen, 2006 folgte Nordkorea. Seit Jahren ist bekannt, dass Israel bis zu 200 Nuklearsprengköpfe besitzt, und die Anreicherungsaktivitäten des Iran stellen ein weiteres ernst zu nehmendes Problem dar. Der bislang verpönte Aufbau eines atomaren Waffenarsenals ist zur politischen Option geworden. Die Sicherheitsstrategien der USA und der EU listen die Verbreitung von Massenvernichtungswaffen ausdrücklich als eine der herausragenden aktuellen und zukünftigen Bedrohungen der globalen Sicherheit auf. Aber auch die anerkannten Nuklearwaffenstaaten unterminieren die Glaubwürdigkeit ihres allgemeinen Abrüstungsversprechens im NVV durch die Weiterentwicklung nationaler Nuklearwaffenstrategien sowie die Modernisierung ihrer Atomwaffensysteme. Diese Entwicklungen und Trends können den NVV untergraben und die Motivation für die Weiterverbreitung von Atomwaffen liefern. Das auslösende Moment kann dabei in regionalen Dynamiken liegen, wie im Falle des Mittleren Ostens, wo davon ausgegangen wird, dass weitere Staaten ein Atomwaffenprogramm in Gang setzen werden, sobald der Iran die entsprechenden Kapazitäten besitzt. Das Beispiel Nordkorea zeigt auf der anderen Seite, dass der Aufbau eines Nuklearwaffenarsenals als wichtiger „bargaining chip" verwendet werden kann, um ein Entgegenkommen wichtiger Staaten oder der internationalen Gemeinschaft insgesamt zu erwirken. ${ }^{2}$

\section{Das neue Strategische Konzept der NATO als Chance}

Bei der Eindämmung der Verbreitung von Nuklearwaffen fällt der NATO eine besondere Rolle zu. Dieser Verantwortung stellte sich die Allianz auch in ihrem Strategischen Konzept von 1999, worin die Fortschritte der Mitgliedstaaten im Bereich der Abrüstung und Rüstungskontrolle dargelegt und weitere Schritte

2 Sebastian Harnisch, Die Krise der Nuklearordnung, SIPO-Reader Online 2008, http://www.readersipo.de. 
ausdrücklich begrüßt werden. ${ }^{3}$ Gleichzeitig werden jene Gefahren und Bedrohungen definiert, die sich aus der Proliferation nuklearer Technologien ergeben.

Das noch immer gültige Strategische Konzept markiert einen wichtigen Meilenstein in der Anpassung der Allianz an das neue Sicherheitsumfeld nach dem Kalten Krieg. Es beschreibt die Rolle der NATO-Nuklearstreitkräfte als „politisch“; dazu bestimmt, Erpressung vorzubeugen und den Frieden zu bewahren. Darüber hinaus konstatiert das Konzept, dass die verminderten Atomwaffenarsenale sowohl zu den Bemühungen beitragen, die Verbreitung von Massenvernichtungswaffen zu vermeiden als auch deren Nutzung zu unterbinden. Die auf ein Minimum reduzierten Nuklearwaffen in Europa seien aber weiterhin unabdingbar, um mögliche Attacken abzuschrecken. ${ }^{4}$ Trotz der Reduzierungen sind in Europa jedoch noch immer Hunderte taktischer Nuklearwaffen der USA und Russlands stationiert.

Die politischen Entwicklungen in der letzten Dekade haben die Proliferationsziele des Jahres 1999 überholt: Die Situation um das Anreicherungsprogramm des Iran hat sich deutlich zugespitzt. ${ }^{5}$ Nordkorea hat 2006 einen Atomwaffentest durchgeführt. Ende 2003 wurde ein weit verzweigtes, von dem „Vater der pakistanischen Atombombe“ Abdul Qadeer Khan organisiertes Netzwerk zur Weitergabe nuklearer Technologie und Expertise an den Iran, an Nordkorea, Libyen und möglicherweise weitere Staaten aufgedeckt. ${ }^{6}$

NATO-Generalsekretär Jaap de Hoop Scheffer hat die Allianz wiederholt dazu aufgefordert, mit der Arbeit an einem neuen Strategischen Konzept zu beginnen. Die Gipfelerklärung von Bukarest 2008 deutet nun darauf hin, dass ein solches Konzept noch im Jubiläumsjahr 2009 oder spätestens 2010 vorgestellt werden könnte: “The Summit [in 2009] will provide an opportunity to further articulate and strengthen the Alliance's vision of its role in meeting the evolving challenges of the 21st century..." Im Zuge dieser strategischen Revision führt die Allianz eine interne Debatte um die zukünftige Rolle nuklearer Abschreckung.

Angesichts dessen stellt sich die Frage, ob ein Beibehalten der gegenwärtigen Nuklearstrategie der NATO, unter Einschluss nuklearer Abschreckung, den aktuellen Herausforderungen gerecht wird oder vielmehr kontraproduktiv wirkt. Im Hinblick auf die NVV-Überprüfungskonferenz 2010, bei der alle Teilnehmer sich um eine Wiederbelebung des globalen Nichtverbreitungsregimes bemühen werden, steht die NATO vor einer großen Aufgabe: mehr Übereinstimmung herzustellen zwischen dem Strategischen Konzept der NATO und den weltweiten Bemühungen um das NVV-Regime. Zwei bedeutende Hindernisse stehen diesem Bestreben im Wege: die Stationierung taktischer Atomwaffen in Europa im Rahmen der nuklearen Teilhabe, die auch als Verletzung des NVV interpretiert

3 NATO Strategic Concept 1999, Par. 19 und 40.

4 Ebenda, Par. 46

5 Der Iran könnte nach Angaben der IAEA schon Anfang 2009 über die erste Atomwaffe verfügen, vgl. Hans Rühle: Eine Bombe zu Weihnachten, in: Süddeutsche Zeitung, 23. Oktober 2008.

6 Monika Heupel, Das A.Q.-Khan-Netzwerk, Transnationale Proliferationsnetzwerke als Herausforderung für die internationale Nichtverbreitungspolitik, SWP-Studie, S 14, Mai 2008

7 NATO Bucharest Summit Declaration, Par. 3 wird ${ }^{8}$, und die derzeit gültige Politik, Atomwaffen gegebenenfalls zuerst einzusetzen.

\section{Nukleare Teilhabe}

Noch 2006 gab die auf Initiative der schwedischen Regierung und der UN gegründete Massenvernichtungswaffen-Kommission (Blix-Kommission) an, dass noch knapp über 400 USamerikanische taktische Nuklearwaffen in Europa stationiert seien. ${ }^{9}$ Hans M. Kristensen, Atomwaffenexperte der Federation of American Scientists, schätzt die Anzahl mittlerweile auf nur noch 150 bis $240 .{ }^{10}$

Die verbleibenden taktischen Waffen sind in europäischen NATO-Mitgliedstaaten stationiert, ein Teil davon im Rahmen der sogenannten nuklearen Teilhabe (nuclear sharing). Demzufolge sind bis zu 140 dieser Waffen zur Nutzung durch Nicht-Atomwaffenstaaten der NATO bestimmt. ${ }^{11}$ Obwohl diese Waffen in Friedenszeiten unter der Obhut der USA verbleiben, können sie in Kriegszeiten den US-Alliierten zur Verfügung gestellt werden. Dies bedeutet, dass im Kriegsfall amerikanische Nuklearwaffen durch deutsche, belgische oder italienische Kampfflugzeuge abgeworfen werden könnten.

Um die Notwendigkeit der nuklearen Teilhabe zu begründen, wird traditionell eine Reihe militärischer und politischer Argumente ins Feld geführt. Die NATO betont insbesondere die politische Dimension. US-amerikanische taktische Nuklearwaffen in Europa werden, erstens, als ein wichtiger „Kitt“ angesehen, um die transatlantischen Beziehungen zu festigen. Das Strategische Konzept der NATO von 1999 besagt: "nuclear forces based in Europe and committed to NATO provide an essential political and military link between the European and the North American members of the Alliance. The Alliance will therefore maintain adequate nuclear forces in Europe."12

Es wird, zweitens, darauf verwiesen, dass die Stationierung taktischer Waffen notwendig sei, um den Verbleib amerikanischer Truppen in Europa zu sichern. Mit der Beibehaltung der taktischen Waffen begegnen die USA somit den Befürchtungen der Alliierten, dass sie ihr Engagement in Europa stark reduzieren würden. Der Blick in Regionen außerhalb Europas zeigt jedoch, dass dies kein zwingender Nexus ist. So hatte der Abzug aller taktischen Atomwaffen aus Japan und Südkorea 1991 keinen Einfluss auf die Präsenz von US-Truppen in diesen Ländern. Auch in Europa folgten dem Abzug der taktischen Nuklearwaffen aus einigen Staaten, wie Griechenland und Großbritannien, keine Truppenverlagerungen.

8 Middle Powers Initiative, Visible Intent. NATO's Responsibility to Nuclear Disarmament, New York, Januar 2008.

9 WMD-Commission: Weapons of Terror: Freeing the World of Nuclear, Biological and Chemical Arms, Stockholm 2006, S. 96.

10 Diese Schätzung basiert auf der Annahme, dass die Atomwaffen aus den US-Luftwaffenstützpunkten in Ramstein (Deutschland) und Lakenheath (Großbritannien) inzwischen abgezogen wurden. Vgl. Hans M. Kristensen: U.S tactical Weapons in Europe: A Review of Post-Cold War Policy, Force Levels, and War Planning, Washington 2008, S.9f. Vgl. zu den aktuellen Zahlen: http://www.fas.org/programs/ssp/nukes/_images/EuroNukes.pdf

11 Ebenda.

12 NATO 1999 Strategic Concept: http://www.nato.int/docu/pr/1999/ p99-065e. htm; dieser Standpunkt wurde im Juni 2007 bestätigt im sogenannten „Final Communiqué", Ministerial meetings of the Defence Planning Committee and the Nuclear Planning Group. 
Ein drittes Argument bezieht sich auf die aus der nuklearen Teilhabe mutmaßlich resultierenden Mitsprache der NATOMitglieder bei der nuklearstrategischen Planung der Allianz sowie bei einem etwaigen Einsatz: ${ }^{13}$ „Die nukleare Teilhabe garantiert für alle Mitgliedstaaten der NATO, dass sie vor einem potenziellen Einsatz von Atomwaffen von den USA konsultiert werden. Dieses System wird daher von keinem unserer Mitgliedstaaten in Frage gestellt. "14 Wenn die Mitsprache mit dem System der nuklearen Teilhabe zusammenhängt, müsste der bislang erfolgte Abzug eines großen Teils der Waffen Besorgnis erregend für Europa sein. Denn implizit bedeutete dies, dass die Teilnehmer des Programms in der nuklearen Planungsgruppe der NATO ein signifikant größeres Gewicht haben als alle anderen nicht (mehr) atomar bewaffneten Mitglieder. Es kann jedoch davon ausgegangen werden, dass es keine grundsätzlich unterschiedlichen Klassen von Mitgliedstaaten in der NATO gibt. Weder im Hinblick auf Mitsprache in der nuklearen Planungsgruppe der NATO, die seit 1979 allen Mitgliedern der Allianz offensteht, noch im Hinblick auf die atomare Abschreckung.

Dies wird am Beispiel Kanadas ersichtlich, das 1989 seine Teilnahme am technischen Teil der nuklearen Teilhabe beendete, ohne dass dem Land dadurch Nachteile in der nuklearen Planungsgruppe entstanden wären. Gleiches gilt für den Ausstieg Griechenlands aus dem Programm 2001 sowie für die neuen NATO-Mitgliedstaaten Osteuropas, die am politischen Programm teilnehmen ohne Atomwaffen auf ihrem Staatsgebiet stationiert zu haben. ${ }^{15}$

\section{Die militärische Dimension}

Neben den politischen Argumenten werden militärische Gründe für die Beibehaltung der nuklearen Teilhabe angeführt: „Die nukleare Teilhabe ist ein notwendiger Teil unserer glaubwürdigen Abschreckung gegenüber potenziellen Angreifern“ betont Guy Roberts, Deputy Assistant Secretary General der NATO. ${ }^{16}$ Dagegen kommt das Science and Technology Committee der Parlamentarischen Versammlung der NATO zu dem Ergebnis, dass die taktischen Nuklearwaffen „nicht maßgeblich zur Sicherheit Europas beitragen" ${ }^{17}$

Die Strategie der nuklearen Teilhabe nahm ihren Anfang in den 1950er Jahren, um US-Alliierte davon abzubringen, eigene Atomwaffenprogramme umzusetzen und sie davon zu überzeugen, dass sie unter dem Schutz des Atomwaffenschirms der USA sicher seien. Diese Funktion nach innen ist nach Ansicht mancher Beobachter auch heute noch aktuell: Die taktischen $\mathrm{Nu}$ klearwaffen der USA auf europäischem Boden (in Verbindung

13 Vgl. die Rede von Karl-Theodor zu Guttenberg im Deutschen Bundestag, abrufbar unter: http://www.cducsu.de/Titel_Rede_Abruestung_angehen_indem_wir_die_NATO_und_die_Strukturen_der_NATO_nutzen/TabID_1/SubTabID_2/InhaltTypID_2/InhaltID_10329/Inhalte.aspx

14 Guy Roberts in einem Interview am 24. Oktober 2008 mit Christos Katsioulis.

15 Die NATO verpflichtete sich in der Gründungsakte des NATO-Russland-Rates zum Verzicht auf die Stationierung von Nuklearwaffen in den neu aufgenommenen Staaten.

16 In einem Interview am 24. Oktober 2008 mit Christos Katsioulis.

17 Science and Technology Committee of the NATO Parliamentary Assembly, Report on Nuclear Weapons Proliferation in 2004, Rapporteur: Claude Nolin, 168 STC 04 E, Brüssel 2004. mit ausgeweiteter Abschreckung der USA) seien die bequemste und am wenigsten provokative Möglichkeit, jene NATO-Staaten von der Lancierung eigener Atomwaffenprogramme abzuhalten, die sich in einer von atomarem Wettrüsten bedrohten Nachbarschaft befinden. Als Beispiel wird der Mittlere Osten mit einem nuklearwaffenfähigen Iran genannt. ${ }^{18}$

In der Frontstellung des Kalten Krieges erhöhte die Verfügungsgewalt etwa Deutschlands über Atomwaffen die Glaubwürdigkeit der nuklearen Abschreckung gegenüber der Sowjetunion. Die Sorge auf westeuropäischer Seite war, dass die USA bei einem konventionellen Angriff der Sowjetunion in Europa nicht mit einem atomaren Gegenschlag antworten würden, der die Vernichtung der USA durch die sowjetischen Atomwaffen riskierte. ${ }^{19}$ Diese Sorge ist im Hinblick auf eine Bedrohung aus dem Mittleren Osten unbegründet. Alle europäischen NATOStaaten befinden sich unter dem nuklearen Schutzschirm der strategischen Atomwaffen der Allianz und die NATO ist auch konventionell jedem potenziellen Angreifer bei Weitem überlegen. Die zusätzliche Verfügbarkeit taktischer Atombomben für Deutschland und andere NATO-Staaten macht daher im heutigen Zusammenhang keinen erkennbaren Unterschied.

Angesichts der begrenzten Reichweite taktischer Waffen und der Tatsache, dass die in den USA stationierten strategischen Waffen jeden potenziellen Ausgangspunkt einer Aggression gegen Europa abdecken, ist der militärische Nutzen einer Stationierung taktischer Atomwaffen in Europa unklar. Vielmehr müssten bei einem Angriff mit taktischen Atombomben - im Gegensatz zum Einsatz strategischer Waffen oder von Cruise Missiles - zusätzliche Schwierigkeiten überwunden werden: Die Bomber müssten bis über das Ziel gelangen und dazu die feindliche Luftabwehr überwinden.

Zudem sind ebenso wie die Nuklearwaffen selbst auch ihre Trägersysteme - hier: Flugzeuge - Modernisierungszyklen unterworfen. Momentan steht in den europäischen Staaten eine Modernisierung der Kampfflugzeuge an. In Deutschland betrifft das die letzte verbliebene Basis mit US-Atomwaffen in Büchel. Die dort bislang von der Bundeswehr eingesetzten Tornados sind in der Lage, nukleare Sprengköpfe abzuwerfen. Sie sollen aber nach 2012 durch den Eurofighter ersetzt werden, der dafür nicht ausgerüstet werden soll. ${ }^{20}$ Eine solche Aufrüstung wäre sehr teuer, so dass Überlegungen zur Weiterführung der nuklearen Teilhabe nicht mehr nur unter verteidigungspolitischen Gesichtspunkten diskutiert werden: „Angesichts des gegebenenfalls notwendigen Wechsels von Flugzeugtypen der europäischen Verbündeten und einer möglicherweise notwendigen Modernisierung der in Europa stationierten B61-Bomben, untersucht die Allianz derzeit verschiedene Möglichkeiten zur Fortsetzung der nuklearen Teilhabe in der Zukunft, wobei haushaltspolitische Gesichtspunkte eine besondere Rolle spielen werden." 21

18 Michael Rühle, Enlightenment in the Second Nuclear Age, in: International Affairs 83 (3), 2007, S. 521.

19 Während des Kalten Krieges war die NATO dem Warschauer Pakt konventionell deutlich unterlegen und daher auf die atomare Abschreckung angewiesen.

20 Deutscher Bundestag, Schriftliche Fragen mit den in der Woche vom 12. Juli eingegangenen Antworten der Bundesregierung, Berlin 2004, S. 27.

21 Guy Roberts in einem Interview am 24. Oktober 2008 mit Christos Katsioulis. 
Es bleibt also unklar, welchen konkreten Sicherheitsvorteil die nukleare Teilhabe bringt. Die Stationierung taktischer Waffen in Europa birgt allerdings Risiken: Erstens bietet sie Moskau einen Vorwand, weiterhin eigene taktische Nuklearwaffen in Westrussland zu stationieren. Zweitens können taktische Nuklearwaffen eher in die Hände von Terroristen gelangen oder unabsichtlich oder absichtlich eingesetzt werden. Die BlixKommission schreibt über taktische Atomwaffen im Vergleich zu strategischen: „[...] they would be easier for outsiders to use, such as a terrorist group. There is a risk of theft or diversion during transport or storage in the field. “22

Drittens liegt eine Gefahr taktischer Nuklearwaffen darin, dass die Sicherheitsstandards für ihre Bewachung und Instandhaltung in Europa offensichtlich nicht den US-amerikanischen Anforderungen genügen. $\mathrm{Zu}$ diesem Schluss kam der Blue Ribbon Review der US Air Force im Februar 2008. ${ }^{23}$ Zwar hat die NATO dem vehement widersprochen und Mängel in der Sicherheit der taktischen Waffen dementiert. Dennoch wird ersichtlich, dass das Konzept der nuklearen Teilhabe zumindest Probleme bei der Kompatibilität der Sicherheitsstandards mit sich bringt. ${ }^{24}$

\section{Die Rolle der Atomwaffen für die Allianz}

Im Gegensatz zu taktischen Nuklearwaffen ist die Bedeutung der strategischen Nuklearwaffen weniger umstritten. Gemäß dem Strategischen Konzept der NATO von 1999 erfüllen die strategischen Nuklearwaffen der USA, Großbritanniens und Frankreichs die Funktion der Abschreckung gegenüber einem potenziellen Angriff auf ein Mitglied der Allianz. Die Debatte über die künftige Nuklearstrategie der NATO schließt aber auch die strategischen Waffen ein. Die Nukleare Planungsgruppe der NATO hat im Juni 2007 Vorschläge willkommen geheißen, die sich mit den „Abschreckungsvoraussetzungen für das 21. Jahrhundert “ 25 befassen. Man darf also eine Einigung der NATO auf eine neue Atomdoktrin erwarten. Aber wie wird diese Doktrin aussehen, und wird sie die Bemühungen um Einigkeit im NVV unterstützen oder schwächen?

Eine Möglichkeit den NVV zu stärken, bestünde in der Annahme einer No-First-Use-Politik, die den Einsatz von Atomwaffen nur als Antwort auf einen atomaren Angriff vorsieht. ${ }^{26}$ Bislang behält sich die NATO das Recht vor, auch als Reaktion auf nichtatomare Angriffe Atomwaffen einzusetzen. Die implizite Drohung, Angriffe mit biologischen oder chemischen Waffen gegebenenfalls mit einem Atomschlag zu beantworten, senkt zwar möglicherweise die Wahrscheinlichkeit solcher Angriffe. Doch mit diesem Vorteil geht der Nachteil einher, dass die Wahrscheinlichkeit eines Einsatzes von Atomwaffen seitens der NATO steigt. Um die Glaubwürdigkeit der Drohung und

22 WMD-Commission (2006), S. 97.

23 Major General Polly A. Peyer (Chair), Air Force Blue Ribbon Review of Nuclear Weapons Policies and Procedures, 8. Februar 2008, http://www.fas.org/nuke/ guide/usa/doctrine/usaf/BRR-2008.pdf, S. 51/52.

24 Vgl. dazu: Oliver Meier, NATO Mulls Nuke Modernization, Security, Arms Control Today, September 2008, http://www.armscontrol.org/print/3311.

25 "Final Communiqué", Ministerialtreffen des Defence Planning Committee und der Nuclear Planning Group, abgehalten in Brüssel am Freitag, 15. Juni 2007.

26 Vgl. dazu: Ivo Daalder, Jan Lodal, The Logic of Zero, in: Foreign Affairs 87(6), 2008, S. 80-95 der politischen Akteure aufrechtzuerhalten, besteht die Gefahr einer „Commitment Trap“: Der Druck auf die Entscheidungsträger steigt, die Drohung im Ernstfall wahr zu machen, auch wenn rein militärisch eine konventionelle Antwort ausreichen würde. ${ }^{27}$

Welche Auswirkungen eine solche Entscheidung zudem auf die militärisch Verantwortlichen hat, wird aus den Erinnerungen von Colin Powell deutlich, der im Zusammenhang mit dem 2. Golfkrieg schildert, wie er den Gedanken an den Einsatz taktischer Nuklearwaffen im Irak kommentierte: „An Atomwaffen sollten wir nicht einmal denken (...) Sie wissen, dass wir diesen Geist nicht aus der Flasche lassen werden. “28

Angesichts der konventionellen Überlegenheit der NATO wiegt der zusätzliche Gewinn an Abschreckung, den diese Strategie bietet, ihren Nachteil nicht auf. Die NATO darf kein Interesse daran haben, einen Präzedenzfall für den Einsatz von Atomwaffen zu schaffen. Eine No-First-Use-Politik würde dieses Risiko vermindern, ohne die Rolle von Atomwaffen als letzte Versicherung gegen existenzielle Bedrohungen durch staatliche Akteure zu beeinträchtigen. Die Wirksamkeit einer atomaren Drohung gegen irrationale Akteure, instabile politische Einheiten und vor allem gegen internationale terroristische Netzwerke ist ohnehin mehr als zweifelhaft.

Es gibt jedoch auch Überlegungen, die in die entgegengesetzte Richtung gehen. Im Januar 2008 veröffentlichten fünf ehemalige hochrangige NATO-Militäroffiziere und -strategen ein Manifest mit dem Titel Towards a Grand Strategy for an Uncertain World. Das Manifest fordert eine strategische Erörterung eines nuklearen Präemptivschlags der NATO als Maßnahme gegen die „unmittelbar bevorstehende“ Verbreitung von Massenvernichtungswaffen. ${ }^{29}$ Dies würde eine klare Absage an die bisherige Politik atomarer Abschreckung bedeuten. Atomare präemptive Vernichtungsschläge als Teil der offiziellen Strategie würden die Hemmschwelle zur Nutzung von Atomwaffen dramatisch senken und die Gefahren der Weiterverbreitung verschärfen. Erstens lieferte diese Politik einen zusätzlichen Anreiz oder zumindest einen Vorwand für dritte Staaten, im Geheimen eigene Atomarsenale aufzubauen um potenzielle Angriffe der NATO abzuschrecken. Sie würde zweitens ein gefährliches Vorbild abgeben, denn andere Atomwaffenstaaten könnten diese Politik übernehmen und einem Staat einen Angriff (ebenfalls mit Atomwaffen oder anderen Massenvernichtungswaffen) androhen, den sie verdächtigen, Massenvernichtungswaffen herzustellen. Inwiefern ein solcher Präemptivschlag in den demokratischen Gesellschaften der Allianz vermittelbar wäre, ist dabei noch gar nicht diskutiert.

Der Umgang mit Staaten, die den NVV nicht befolgen, erfordert vielmehr individuelle Lösungen, die auf die Interessenlage der jeweiligen Staaten und der Region sowie auf die jeweiligen Kapazitäten ausgerichtet sind. ${ }^{30}$ Paketvereinbarungen wie im Falle Libyens oder Nordkoreas versprechen mehr Erfolg als

27 Scott Sagan diskutiert dieses Problem ausführlich anhand der US-Strategie der "calculated ambiguity": Scott D. Sagan; The Commitment Trap, in: International Security 24 (4), Spring 2000: 85-115.

28 Colin Powell, Mein Weg, Berlin 2005, S. 500.

29 Klaus Naumann u.a., Towards a Grand Strategy for an Uncertain World, Lunteren 2007.

30 Michael Rühle (2007), S. 520. 
die Androhung eines präemptiven Nuklearschlags, indem sie politische, ökonomische und Sicherheitsinstrumente als Zuckerbrot und Peitsche kombinieren. Dies schließt den Einsatz (konventioneller) militärischer Gewalt bzw. die Drohung damit nicht aus. Diese Drohung ist insofern wirkungsvoll, als die NATO über die weltweit am höchsten entwickelte und stärkste konventionelle Streitkraft verfügt. Dies ist ausreichend als „Peitsche“ gegenüber potenziellen Proliferatoren.

Die präemptive Anwendung atomarer Waffen würde schließlich zwei der unmittelbarsten Bedrohungen des ,zweiten Atomzeitalters" nicht gerecht: Weder der Nuklearterrorismus noch die Verbreitung von Nuklearwaffentechnologie durch transnationale Netzwerke lassen sich mit den Mitteln der Abschreckung bekämpfen. Stattdessen hat die Staatengemeinschaft, basierend auf der UN-SR-Resolution 1540 sowie auf der von den USA initiierten Proliferation Security Initiative ein leistungsfähiges Instrumentarium der „Counter-Proliferation“ aufgebaut, das diesen Herausforderungen effektiv begegnen kann.

\section{Die Nuklearwaffen der NATO - welches ist der richtige Kurs?}

Zur Zeit sind die USA die einzige Atommacht, die weiterhin Atomwaffen außerhalb ihres eigenen Territoriums stationiert: die 150 bis 240 taktischen Waffen in Europa. Das System der nuklearen Teilhabe und die vor allem politisch definierte Rolle der Nuklearwaffen für das Bündnis hindern die NATO bislang daran, diese Strategie grundlegend zu überdenken. Das neue Strategische Konzept, das vermutlich im Schlüsseljahr der Nichtverbreitung 2010 vorgestellt wird, bietet die Chance, diesen Themenkomplex neu zu diskutieren.

Die Signale aus der NATO sind bislang wenig ermutigend. Zwar empfiehlt der Berichterstatter der Parlamentarischen Versammlung der NATO, Pierre Claude Nolin, der Allianz, die Möglichkeit in Betracht zu ziehen, die taktischen Waffen aus Europa abzuziehen. Er nennt dafür zwei Vorbedingungen: a) die europäischen Alliierten erhalten die Infrastruktur und ihre an Nuklearwaffen ausgebildeten Einheiten, so dass die Praxis der Teilhabe wenn notwendig wieder aufgenommen werden kann; b) Russland willigt ein, reziprok dazu seine taktischen Waffen außer Dienst zu stellen und nachprüfbar zu sichern. ${ }^{31}$ Dem gegenüber steht jedoch die Aussage von Guy Roberts, dass es unwahrscheinlich sei, dass die NATO fundamentale Änderungen an ihrer Nuklearwaffenpolitik vornehmen wird. ${ }^{32}$

Im breiteren internationalen Kontext ist hingegen Bewegung in die Diskussion um Nuklearwaffen im 21. Jahrhundert gekommen. Angestoßen von einem gemeinsamen Aufruf der ehemaligen US-amerikanischen Außenminister Kissinger und Shultz, des ehemaligen US-Verteidigungsministers Perry und des US-Senators Nunn zur Abschaffung aller Atomwaffen ${ }^{33}$ hat

31 Committee Report to the NATO Parliamentary Assembly, Reducing Global Nuclear Threats: Contribution of Official Nuclear Weapon States, Rapporteur: Pierre Claude Nolin, 167 STC 08 E, Brüssel 2008; dieser Bericht spiegelt bislang nur die Meinung des Berichterstatters wieder, weil er von der Parlamentarischen Versammlung noch nicht angenommen wurde.

32 Oliver Meier (2008).

33 George P. Shultz, William J. Perry, Henry A. Kissinger and Sam Nunn: Toward a Nuclear-Free World. The Wall Street Journal, January 4, 2007. sich zunächst in Washingtoner sicherheitspolitischen Kreisen der Gedanke ausgebreitet, dass ein neuer, radikalerer Ansatz zur Verhinderung einer massiven Proliferation von Atomwaffen notwendig ist. Dieser Ansatz hat internationalen Widerhall gefunden: u.a. in einem ähnlich lautenden Artikel ehemaliger britischer Außenminister zusammen mit dem früheren NATOGeneralsekretär Robertson, in der aktuellen Literatur und in einer Kampagne mit prominenter Unterstützung aus dem internationalen sicherheitspolitischen Umfeld. ${ }^{34}$ Die Erarbeitung eines neuen Strategischen Konzepts bietet auch der NATO die Chance zur Neuausrichtung ihrer Nuklearwaffen-Politik .

Der Amtsantritt der neuen US-Administration Anfang 2009 und die bevorstehenden Verhandlungen zur Überprüfung des NVV bieten ein ideales Zeitfenster für die NATO, um die Rolle der taktischen Nuklearwaffen zu überdenken. Die NATO sollte daher die Möglichkeit ergreifen, bei der Neufassung ihres Strategischen Konzepts die Funktion der Nuklearwaffen grundlegend zu überdenken. Ein neues Strategisches Konzept, das einen konkreten Fahrplan hin zur zero option ${ }^{35}$ taktischer Nuklearwaffen in Europa und zu einer no-first-use option festschreibt, könnte der Katalysator einer effektiveren und glaubwürdigeren NATOAbrüstungspolitik sein.

Die Neufassung des Strategischen Konzeptes unter Berücksichtigung dieser beiden Maßgaben birgt mehrere strategische Vorteile für die NATO:

- Die Sicherheit der Mitgliedstaaten wäre weiterhin gewährleistet, da sie unter dem nuklearen Abschreckungsschirm durch die strategischen Waffen der USA geschützt sind;

- die teure Aufrüstung der neuen Generation von Flugzeugen ${ }^{36}$ wäre hinfällig und würde die ohnehin strapazierten Haushalte entlasten;

- die angebliche politische Kohäsionsfunktion der nuklearen Teilhabe würde entfallen und die Mitgliedstaaten in eine überfällige Debatte über die gemeinsamen Ziele und Werte zwingen;

- die völkerrechtlich zumindest fragwürdige Praxis der nuklearen Teilhabe wäre beendet; ein zentrales Argument gegen die USA und ihre Alliierten im Kontext des NVV würde entfallen;

- Russland könnte mit Blick auf diese Festlegung zu Zugeständnissen hinsichtlich seiner eigenen taktischen Nuklearwaffen gedrängt werden;

- die Glaubwürdigkeit der NATO-Mitgliedstaaten und insbesondere der USA wären im Hinblick auf die Verhandlungen zum NVV gestärkt, weil die Allianz ein klares Abrüstungssignal setzen würde.

34 Douglas Hurd, Malcolm Rifkind, David Owen and George Robertson: Start worrying and learn to ditch the bomb, The Times, 30 June 2008; George Perkovich and James M. Acton: Abolishing Nuclear Weapons. Adelphi Papers 396, International Institute For Strategic Studies, September 2008; www.globalzero.org

35 Rolf Mützenich, The Undiminished Relevance of Disarmament and Arms Control. Ten Theses, International Policy Analysis, Friedrich-Ebert-Stiftung, May 2008, S. 2.

36 Die gegenwärtig von der Luftwaffe für den nuklearen Einsatz vorgesehenen MRCA Tornado sollen längstens bis 2020 im Dienst sein. 